\title{
LUPUS SCIENCE\& MEDICINE \\ Engaging African ancestry participants in SLE clinical trials
}

To cite: Anjorin A, Lipsky P. Engaging African ancestry participants in SLE clinical trials. Lupus Science \& Medicine 2018;5:e000297. doi:10.1136/ lupus-2018-000297

Received 26 July 2018 Revised 19 October 2018 Accepted 25 October 2018
Check for updates

(C) Author(s) (or their employer(s)) 2018. Re-use permitted under CC BY-NC. No commercial re-use. See rights and permissions. Published by BMJ.

${ }^{1}$ RILITE Research Institute, and University of Virginia, Charlottesville, Virginia, USA ${ }^{2}$ RILITE Research Institute, Charlottesville, Virginia, USA

Correspondence to

Dr Peter Lipsky; peterlipsky@ comcast.net

\section{ABSTRACT}

SLE is a complex autoimmune disease with genetic and clinical differences between patients that appear to reside along ancestral lines. Over the last 20 years, a preponderance of evidence has shown that SLE is more common and severe in minority populations, particularly in African ancestry (AA) women. However, in clinical trials for new therapies of SLE, AA is often under-represented. Without enrolling sufficient AA participants, it is difficult to ascertain the safety and efficacy of new potential therapies among individuals with SLE of different ancestries. Although enrolling minority populations in clinical trials has been a significant challenge for many reasons, the various stakeholders involved in clinical research could act within their own realms to develop new paradigms and policies to bolster the inclusion of $A A$ in the development of new therapies.

\section{INTRODUCTION}

SLE is a chronic autoimmune disease that affects multiple organ systems. ${ }^{1}$ Several factors contribute to the development and severity of SLE, including genetics, epigenetics and environmental stimuli. Both innate and adaptive immune system dysfunction contribute to the pathogenesis of SLE, which leads to inflammation and tissue damage. There is currently no cure for SLE, but common therapies include glucocorticoids, antimalarials and immunosuppressive drugs. ${ }^{2}$ Stakeholders agree that safer and more efficacious medications are needed to treat persons with SLE more effectively.

SLE is more common and severe in minority populations. ${ }^{3} \quad$ Individuals from minority populations experience more abrupt disease onset, a higher degree of disease activity and greater severity of clinical manifestations. ${ }^{3}$ Despite the disparity in disease manifestations and severity in minority individuals, clinical trials in SLE have usually recruited insufficient minority participants, especially African ancestry (AA) subjects, to determine whether medications are effective in this population. The result is that the benefits of a medication in AA subjects are often not fully evaluated. Moreover, since there are important differences in the genetics, biology and clinical impact of SLE in persons of different ancestries, it cannot be inferred that benefits in European ancestry (EA) individuals will predict efficacy and safety in patients with lupus of African ancestry.

In this review, we sought to (1) summarise the extensive body of literature demonstrating differences in genetics and health impact in AA patients with SLE compared with EA counterparts, and (2) describe challenges in recruiting AA for clinical trials. Although it is indisputable that social determinants of health, such as education and food insecurity, influence health outcomes, the conceptual model applied here emphasises the impact of genetic and biological differences in disease pathogenesis in AA and EA patients with SLE. The literature provides overwhelming evidence that SLE is more common and severe in AA, providing empirical support to bolster enrolment of AA participants in clinical trials for new therapies for lupus. We conclude with a discussion of relevant stakeholders in clinical research who could be involved in developing policies or protocols that promote inclusion of AA participants in trials.

Of note, other ancestral groups are discussed only when relevant information is available. Hispanics, Asian-Americans and American-Indian/Alaskans are also under-represented in clinical trials in the USA; however, because of gaps in the literature and the desire to focus this review, these minority populations are not fully discussed. Furthermore, in this work, we focus primarily on under-representation of AA in SLE clinical trials. AA under-representation in clinical trials for other diseases such as cancer ${ }^{4}$ and cardiovascular disease $(\mathrm{CVD})^{5}$ has been well documented and it is not discussed within the scope of this review.

\section{The impact of ancestry on lupus manifestations}

SLE is most prevalent in AA persons, and some studies have suggested that there may be different genetic components contributing to disease development and progression in minority populations. ${ }^{3}$ Importantly, 
in a transancestral genetic mapping study of SLE, Langefeld et al found that there is a complex, multigenic effect in SLE, suggesting a strong heterogeneous genetic component to disease activity that differs according to ancestral background. ${ }^{6}$ This has implied a 'racial' component to lupus pathogenesis. 'Race' as conceptualised today, however, is an imperfect surrogate for understanding genetic variation among individuals with different ancestral backgrounds. ${ }^{6}$ Racial categories can be rigid, which ultimately limit the ability to appreciate underlying genetic variability stemming from a multiplicity of ancestral lineages. Despite this, in SLE there are clear genetic differences between patients that appear to exist along ancestral lines.

It is important here to make a distinction between race and ethnicity. While the definition of both words has been the subject of contentious debate, ethnicity is believed to be self-identified or societally identified, and more dynamic because it encompasses factors such as geographical origin, migratory status, housing and employment patterns, cultural factors, as well as genetic ancestry. ${ }^{6}$ Like racial categories, ethnic boundaries are not fixed, and there still remains the potential for significant variation between individuals within a defined group. In individuals with SLE, differences in ancestral backgrounds may play a role in the pathogenesis, susceptibility, organ involvement and response to medications, whereas ethnicity may contribute to severity, outcomes and attitudes towards subjects, such as clinical research participation.

In addition to ancestry, there are several other factors known as social determinants of health that have been associated with health outcomes. These determinants include, but are not limited to, educational attainment, employment status and food insecurity. ${ }^{7}$ Disentangling the impact of determinants is complex as they tend to intersect and interact with one another. As such, it is important that we acknowledge that ancestry is not the singular factor influencing SLE disease manifestations and patient enrolment in clinical trials. However, the overwhelming burden of SLE on minority individuals, specifically AA subjects, along with the markedly disparate genetics, makes ancestry an appropriate topic of focus.

\section{METHODS}

Several lines of evidence indicate that there are substantial differences in lupus based on ancestral background. To gain a comprehensive understanding of these differences, we conducted a systematic literature review of published studies in English between June 1987 and June 2017 using PubMed MEDLINE. This choice was made to reflect both older and more current ascertainment of disease outcomes and response to medications for SLE. The search strategy was as follows: (race AND lupus) AND (("1987/06/01" (PDat): “2017/06/30" (PDat)) AND Humans (Mesh) AND English (lang)).
Studies were included in the systematic literature review based on the following criteria: studies: all reports on SLE outcomes or drug effects described according to different racial/ethnic groups; patients: all adult patients ( $\geq 18$ years) regardless of disease characteristics or organ manifestations; and outcomes: mortality rates, survival rates, clinical manifestations and disease activity according to racial/ethnic groups. Studies not published in English, published before 1987, reviews, conference proceedings, case reports, letters and commentaries were excluded. Additionally, genetic or immunological papers were not included. Less generalisable studies conducted in small ethnic groups were not included (ie, Han Chinese, or black South Africans). Studies performed in paediatric patients were also excluded.

As of July 2017, 1646 articles were extracted by this search procedure. After review of the titles, 154 relevant publications were identified and assessed for eligibility. On reviewing the abstracts, 62 publications met the inclusion criteria and were retrieved for full-text review. Two studies on incidence and prevalence of SLE published after the initial search procedure in July 2017 were included. Because of the lack of relevant data, a separate review was conducted to assess differences in medication response. In total, 59 publications were ultimately included in the literature review.

\section{RESULTS AND DISCUSSION \\ Differences between $A A$ and EA lupus \\ Genetics}

A large genome-wide association study interrogating the association of lupus with genetic regions identified by Immunochip genotyping has emphasised the differences between genetic associations in EA and AA subjects. ${ }^{8}$ Many of the 58 genetic regions associated with SLE in EA were not identified in AA subjects, and a few of the 9 genetic regions associated with SLE in AA were not found in EA patients. Importantly, when the cumulative impact of all the EA-associated genetic regions was considered in EA subjects, the OR for lupus was nearly 30, whereas when the same EA-associated genetic regions were considered in AA patients, the OR for lupus was only approximately 3 . The findings emphasise the major differences in the genetic association with lupus in EA versus AA patients.

\section{Incidence and prevalence}

In recent years, four studies have comprehensively measured the incidence and prevalence of SLE in different cohorts within the USA with great consistency. Detailed case finding and verification in four regions of the USA have reported similar results, with the incidence varying between 4.6 and 5.6/100 000 person-years and the prevalence varying between 62.2 and 84.8/100 $000 .^{9-12}$ In all studies, the incidence and prevalence in AA women greatly exceeded those in white women by a factor of 
2-6. ${ }^{9-12}$ Accounting for the percentage of AA in the US adult population, this results in as many as $30 \%-43 \%$ of patients with SLE in the USA being of African ancestry. ${ }^{13}$

\section{Nephropathy}

Lupus nephritis (LN) occurs more frequently in persons from African, Asian and Hispanic ancestral background compared with Caucasians. ${ }^{14}$ In the US Medicaid population of patients with SLE from 2000 to 2004, the estimated prevalence of $\mathrm{LN}$ was about four times higher in AA (59.69/100 000) compared with EA subjects $(15.83 / 100000),{ }^{15}$ as was reported previously: AA $36.7 \%-52 \%$ versus EA $18.8 \%-40 \% .{ }^{9-12}$ In the LUMINA (Lupus in Minorities: Nature vs Nurture) cohort, AA (HR 2.57; $\mathrm{p}=0.0097)$ and Hispanic ancestry $(\mathrm{HR}=2.32$; $\mathrm{p}=0.0430$ ) were associated with shorter time to $\mathrm{LN}$ occurrence compared with the EA reference group. ${ }^{16}$ Notably, one cross-sectional study of persons with SLE employed genotyping to show that European genetic ancestry may be protective against the development of $\mathrm{LN}$ as a $10 \%$ increase in the proportion of EA was associated with a $15 \%$ reduction in the odds of developing a renal disease. ${ }^{17}$

In addition, a greater proportion of AA patients with SLE developed end-stage renal disease (ESRD, $8.4 \%-15.3 \%)$ compared with EA counterparts $(1.2 \%-$ $4.5 \%),{ }^{9} 10$ whereas more AA patients with severe LN progressed to ESRD than EA patients with $\mathrm{LN},{ }^{18-20}$ and AA subjects with LN-caused ESRD had a significantly increased risk of death compared with other ethnicities $(\mathrm{HR}=1.18 ; 95 \%$ CI 1.11 to 1.25$) .{ }^{21}$ In support of this finding, Gómez-Puerta $e t a l^{22}$ found similar results showing that AA patients with LN-associated ESRD have an increased mortality risk compared with EA patients (HR $=1.27 ; 95 \%$ CI 1.18 to 1.36$)$. Additionally they found that after adjusting for comorbidities, AA patients were $66 \%$ more likely to be admitted for haemorrhagic stroke, and they had a $35 \%$ higher risk of admission for heart failure than EA patients with LN-associated ESRD. ${ }^{22}$

\section{Cardiovascular disease}

In a study of patients with SLE enrolled in Medicaid, AA patients were found to have the highest CVD event rates ( $\mathrm{HR}=10.57 ; 95 \%$ CI 9.96 to 11.22$)$ and greater risk of CVD events (HR=1.14; 95\% CI 1.03 to 1.26 ) compared with Hispanics, EA subjects and Asians. ${ }^{23}$ Similarly, AA women with SLE were twice as likely to develop carotid plaques than EA women (43.5\% vs $29.6 \%$; OR 1.94 , 95\% CI 1.03 to 3.65$).{ }^{24}$ Moreover, higher blood pressure, increased body mass index and lipoprotein abnormalities were more prevalent in AA women. Finally, AA patients were found to have more lupus-related risk factors for CVD, such as greater disease activity and more corticosteroid use for longer periods of time. ${ }^{24}$ The combination of these factors might explain the ancestral differences in plaque burden. ${ }^{24}$ However, Scalzi $e t$ $a l^{25}$ found that after adjusting for CVD risk factors, such as associated comorbidities, insurance, medications and myocardial infarction characteristics, AA was associated with higher mortality at younger ages.

\section{Disease activity and damage accrual}

Levels of disease activity and damage accrual have also been shown to vary according to ancestral background. González et al and others have found that ethnic minorities developed SLE more frequently and they tended to have more acute onset of disease activity and more damage accrual compared with EA subjects. ${ }^{19} 26$ They typically presented with more clinical manifestations that were greater in severity. In non-EA subjects, SLE tended to present at a younger age with more rapid accumulation of damage ${ }^{192728}$ and higher mortality rates. ${ }^{26}$

Bruce $e t a l^{29}$ have shown in the Systemic Lupus International Collaborating Clinics Inception Cohort that American patients of African ancestry had a higher risk of transitioning from no damage to higher damage than whites in Europe or Canada (AA relative transition rates (RTR) 2.55 ; $95 \%$ CI 1.92 to 3.40 ; EA RTR 1.99 ; $95 \%$ CI 1.33 to 2.96). Furthermore, Cooper $e t a l^{30}$ found that the extent of organ damage among recently diagnosed patients with SLE was greater in AA patients, and the prevalence of skin damage, diabetes and alopecia was significantly greater in AA patients compared with EA $($ ORs $\geq 5) .{ }^{30}$ In the Carolina Lupus Study, AA patients were more likely to have discoid lupus and proteinuria. Additionally, anti-Sm and anti-ribonuclear protein autoantibodies were observed more frequently in AA (ORs $\geq 3) .{ }^{27}$

\section{Medication response}

Cyclophosphamide, a common therapy for LN, has been shown to have differential effects according to ancestral background. In a study assessing renal outcomes of cyclophosphamide therapy for SLE diffuse proliferative lupus glomerulonephritis (SLE-DPGN), Dooley et $a l$ found that renal survival was significantly $(\mathrm{p}=0.02)$ worse among AA patients compared with non-AA subjects. ${ }^{31}$ EA patients with SLE-DPGN retained 95\% renal function at year 5, whereas AA patients displayed progressive yearly decline in renal function, with just $58 \%$ retaining renal function at year $5 .{ }^{31}$

Another common therapy for LN, mycophenolate mofetil (MMF), also shows differences in treatment effects related to ancestry. In a study comparing the effects of intravenous cyclophosphamide (IVC) and MMF, Appel et $a l^{32}$ found no significant differences. However, they did find a statistically significant association between treatment group and ancestry $(p=0.047)$. More patients in the non-white, non-Asian subgroup responded better to MMF than IVC, and this effect was not explained by baseline differences in disease characteristics. ${ }^{32}$

Belimumab, the only new therapy approved for lupus in the past 60 years, is a monoclonal antibody that 
inhibits the activity of B lymphocyte stimulator, elevated levels of which are associated with SLE disease activity. ${ }^{33}$ When assessed with the Systemic Lupus Erythematosus Responder Index, belimumab administered with standard of care treatment was shown to have a significantly higher response rate than standard of care treatment and placebo at 52 weeks $(\mathrm{p} \leq 0.017) .{ }^{33}{ }^{34}$ However, in both the Belimumab in Subjects with SLE (BLISS)-52 and BLISS-76 pivotal studies combined, only 148 out of 1648 trial participants (9\%) were AA. ${ }^{33}$ With such low enrolment, subgroup analyses in this trial were underpowered and inconclusive, but suggested that the agent was not effective in AA participants. Another study was conducted with a subcutaneous formulation of belimumab with approximately $11 \%$ AA participants enrolled, and these results, similarly, did not show a statistically significant treatment effect in AA. ${ }^{35}$

\section{Survival and mortality}

AA subjects with SLE have been shown to experience increased mortality rates compared with EA patients. ${ }^{36-40}$ Korbet $e t a l^{20}$ found that renal survival and patient survival (with and without ESRD) were significantly $(\mathrm{p} \leq 0.045)$ worse in AA patients compared with EA subjects. Specifically, they found that the median patient survival without ESRD was 182 months for EA patients compared with 40 months for AA patients. ${ }^{20}$ Furthermore, Ward et $a t^{41}$ showed that survival was significantly worse for AA patients at 5,10 and 15 years compared with EA patients with SLE $(\mathrm{p}=0.005)$. For EA patients, the 5-year, 10-year and 15-year survival estimates were $87 \%, 76 \%$ and $70 \%$ compared with $76 \%$, $65 \%$ and $55 \%$ for AA patients. ${ }^{41}$

\section{Socioeconomic status}

In addition to ancestry, socioeconomic status (SES) has also been shown to influence disease outcome. ${ }^{41}$ However, in the UK Lupus Cohort conducted primarily in the UK system, where healthcare is available at point of delivery, the frequency of renal failure in patients with SLE remained greater among AA than EA patients $(\mathrm{p}=0.003) .{ }^{42}$ In the USA, several studies conducted among Medicaid beneficiaries also found greater severity of disease among minority patients. ${ }^{15} 3643$ These findings suggest that ancestral background may be more impactful than SES in predicting SLE disease outcomes.

\section{Summary}

The data support the conclusion that many aspects of SLE are different in AA individuals and raise the question as to whether SLE is even the same disease in people of different ancestries. Importantly, without appropriate representation of minority participants in clinical trials, it is challenging to ascertain the true efficacy of therapies for SLE in AA individuals. There is sufficient evidence suggesting that drug effects may differ according to ancestry, ${ }^{32-35} 44$ and because SLE predominantly affects minority populations ${ }^{9} 1045$ there is a scientific imperative to address this concern.

\section{Challenges in SLE clinical trials}

Currently in the USA, AA makes up $12 \%$ of the overall population. ${ }^{46}$ However, analysis of recent prevalence studies ${ }^{913}$ indicates that AA may represent as much as $30 \%-43 \%$ of all patients with SLE in the USA. Despite this disparate burden of disease affecting AA patients, patterns of under-representation are observable in clinical trials for SLE therapies, as evidenced by the trials investigating the safety and efficacy of belimumab. ${ }^{33}$

Enrolling minority patients in trials has been a significant challenge for several reasons. ${ }^{47} 48$ In a systematic review, George et $a t^{49}$ reported that barriers across minority groups to trial participation include mistrust, competing demands, lack of access to information, stigma, health insurance coverage and legal status in the USA. Particularly among AA, mistrust is thought to stem from the legacy of the infamous US Public Health Service Tuskegee Syphilis studies from 1932 to $1972 .^{50}$ However, in a study of reported consent rates by race and ethnicity of more than 70000 individuals, Wendler et $a \tilde{l}^{51}$ found minimal differences in the rate at which minorities and non-Hispanic whites agree to participate in health research. When eligible and invited to participate in studies, AA subjects were as willing to participate, and in some cases more willing to participate, in health research than non-Hispanic whites. ${ }^{51}$ Although mistrust likely exists in minority communities, the results of this study provide strong evidence that it may not have a major impact on low participation of minority patients in clinical trials.

Besides possible mistrust on the part of minority participants, studies have shown that some physicians may have implicit biases about AA subjects which could deter them from approaching AA individuals to participate in clinical trials. ${ }^{52-55}$ Specifically, Moskowitz et a $\tilde{l}^{53}$ found that primary care physicians are less trusting of minority patients than of white patients. ${ }^{53}$ Other studies have reported that physicians with more implicit biases may perceive minority patients as less likely to comply with recommended therapies, ${ }^{56}$ and racial stereotypes have been shown to influence physicians' diagnoses and treatment recommendations of AA patients. ${ }^{54}$ The likelihood that a patient will adhere to recommended therapies is a common consideration among physicians when enrolling patients in clinical trials. ${ }^{56}$ In a study assessing factors associated with differences in morbidity observed between AA and EA patients with SLE, Petri $e t$ $a \tilde{l}^{\tilde{7}}$ found that physicians rated AA patients as less globally adherent than whites $(66.3 \%$ vs $43.5 \%$; OR $=0.39$, $95 \%$ CI 0.22 to 0.70 ), although there was no significant difference in the average percentage of routine protocol visits kept by AA and EA patients. ${ }^{57}$ This misperception in differences in adherence between EA and AA patients could stem from the belief that AA patients experience more barriers to adherence than their EA counterparts. 
Together these findings suggest that physicians may be less likely to consider minority patients as participants in clinical trials, ultimately limiting their access and opportunities for inclusion. ${ }^{58} 59$

Efforts to increase minority patient participation could focus on the complex relationship between the doctor and the patient, with emphasis on dispelling the mistrust and biases that can influence effective recruiting. ${ }^{515859}$ Because most patients learn about clinical trials through their physician, it is important to consider patient-physician relationships as an opportunity to improve access for minority patients. It has been well established that sociocultural differences between the patient and the physician can influence clinical decision-making and communication. ${ }^{60-63}$ To recruit minority participants for clinical trials effectively, it is imperative to overcome challenges in communication and decision-making associated with sociocultural differences by acknowledging implicit biases and working to deconstruct them in patient interactions. ${ }^{64}$ Specifically, efforts to incorporate implicit biases training into undergraduate, graduate and continuing medical education could yield positive outcomes for trainees, physicians and patients alike. Moreover, training on implicit bias or certification that such training has occurred could be included as part of site initiation for participation in clinical trials.

Another approach could focus on improving ethnic concordance in the patient-physician relationship. ${ }^{65}$ Ethnic concordance has been associated with more positive interactions, ${ }^{61}{ }^{66}$ in part, because of greater perceived similarity $^{62}$ and stronger communication facilitated by more participatory decision-making styles. ${ }^{61}$ Through qualitative focus group interviews with ethnically diverse patients, García $e t a b^{66}$ found that patients have clear preferences for ethnically concordant rather than discordant primary care physicians, who they believed could offer them better healthcare. ${ }^{66}$

These findings are consistent with other studies that have shown that AA and Hispanic physicians are more likely to care for minority patients in poorer areas than non-Hispanic white physicians. ${ }^{67}$ These results have significant implications for improving access to clinical trials for minority patients. Agodoa et a $\tilde{\nu}^{0}$ suggested that the limiting factor to minority patient participation in clinical trials may be related to the lack of access to clinical trials for minority physicians. Many clinical trials are carried out in academic medical centres (AMCs) or predominantly white private practices. ${ }^{68}$ However, minority physicians are more likely to establish their practices in underserved communities, ${ }^{67}$ which limits their involvement with clinical research occurring at AMCs or in more affluent areas. To make progress towards alleviating this barrier to participation, it is important to identify important clinical care dynamics and stakeholders who could be influential in developing effective solutions.
Minority participants

A common misconception in clinical research is that minority individuals are less willing to participate in trials primarily because of mistrust of the medical community rooted in knowledge of the Tuskegee Syphilis experiments, a seminal event among numerous cases of research misconduct towards minority participants. Despite potentially harboured mistrust, it does not appear to be a limiting factor in patient participation. ${ }^{5051}$

To provide further insight into this subject, Mosley-Williams et $a l^{69}$ conducted a study assessing 19 factors previously indicated as barriers to treatment adherence among AA and white women with SLE. They found that AA patients were no more mistrusting of their physicians or hospitals than were white patients. Ethnic groups were comparable in views of their physician's skill and style, level of depression, SLE signs and symptoms, perception of treatment efficacy, need for child care, and social support. Additionally, their results showed that the ethnic groups did not differ in regard to compliance with medications, whereas white patients showed slightly lower adherence for keeping clinic appointments. ${ }^{69}$ These findings call into question the validity of the supposition that mistrust ${ }^{70}$ or challenges with adherence are significant factors in determining a patient's willingness to participate in clinical trials. Moreover, the findings challenge some of the physician biases that may interfere with minority enrolment in trials.

Katz et $a l^{71}$ carried out an additional analysis of factors that influence patients' willingness to participate in biomedical research through the Tuskegee Legacy Project (TLP). Their results indicated that while AA are 1.8 times as likely as EA subjects to have a greater fear of participating in biomedical research, they are nonetheless as willing to participate, particularly when the study was run by their own physician or an AMC. ${ }^{71}$ The conclusions of the TLP study are consistent with the literature on the impact of the patient-physician relationship in influencing health behaviours and outcomes, ${ }^{62}$ and indicate that the patient-physician relationship is an important mechanism to inform minority patients of ongoing clinical research. In addition, public education on the nature of clinical trials and the inherent protections built into clinical trials could help address the knowledge gap and also the residual mistrust of the clinical trial process. Such an educational effort could influence attitudes about clinical trial participation and simplify the discussion between physicians and prospective trial participants.

\section{Community-based medicine}

Community-based physicians can be essential in increasing the enrolment of minority patients into clinical trials, especially those physicians from an ethnic minority background as they tend to care for a disproportionately large number of minority patients. ${ }^{67}$ As such, community-based 
practices can serve as an important source of minority participants for clinical trials. However, minority physicians practising in communities are poorly represented in clinical research because of several constraints. Since AA and Hispanic physicians often practise in areas with fewer physicians per capita ${ }^{67}$ they usually have a greater patient load. Additionally, AA physicians care for more patients insured by providers with lower reimbursement rates such as Medicaid than other physicians, and Hispanic physicians care for more uninsured patients than physicians from other ethnic groups. ${ }^{67}$

The conditions in which these physicians conduct their medical practice can make it challenging to participate in clinical trials that are time-intensive and resource-intensive. These barriers to participation in clinical research are not easily surmountable. A potential solution could create an opportunity for community-based physicians to engage, without requiring their practices to undergo expensive, arduous infrastructural changes, through a partnership with physicians at local established clinical trial sites that are already equipped with the research coordinators, special equipment and protocols for conducting trials. To improve the enrolment of minority participants in clinical trials, the development of innovative pathways to facilitate knowledge sharing and novel partnerships between clinical investigators and community-based physicians could be considered. ${ }^{65}$ Funding opportunities encouraging collaborations between academic and community medical providers could serve as an incentive for engaging a greater number of minority subjects in SLE clinical research. Moreover, the pharmaceutical companies and contract research organisations (CROs) organising trials could require such collaborations and/ or reward their establishment.

\section{Academic-based medicine}

Many clinical trials in SLE are conducted in AMCs. Within an AMC, clinical investigators work with CROs in established partnerships to organise and execute trials frequently sponsored by pharmaceutical companies. Even though many AMCs care for large numbers of minority individuals because of their commitment to provide care for the underinsured, the rates of recruitment of minority subjects in AMC clinical trials remain suboptimal. ${ }^{59} 72$ This could be associated with issues related to known barriers to clinical trial participation, such as patient trust, physician implicit bias, as well as a variety of other aspects of the function of AMC. ${ }^{46555}$ Overcoming some of these internal challenges could increase minority participation in clinical trials since these changes would not require major changes in the operation of the healthcare system.

Another possible way to overcome the current challenges in minority participant recruitment in clinical trials could be the development of collaborations with local community practices that care for minority patients and may have more effective communication with these individuals. ${ }^{46}{ }^{73}$ Although there have been limited studies exploring this approach in lupus clinical trials, the
National Cancer Institute (NCI) funds more than 10 clinical cooperative oncology groups. While the headquarters for these groups are usually located at major AMCs, the majority of patient recruitment to cancer clinical trials is derived from community-based oncologists. ${ }^{74}$ Additionally, to support efforts to involve AA and other minority subjects in clinical trials within the institutions in which they receive care, NCI sponsors the Community Clinical Oncology Program and the Minority-Based Community Clinical Oncology Program. ${ }^{74}$

Furthermore, in response to the small percentage of minority physicians in oncology divisions in AMCs, the Society of Cancer Researchers Advocating Therapeutic Excellence for Special Populations (SOCRATES) was developed. SOCRATES is composed of African-American clinical oncologists who are members of the National Medical Association, the American Society for Therapeutic Radiology and Oncology, and other major cooperative cancer clinical trial groups. ${ }^{65}$ These cooperative groups in oncology serve as practical models for engaging clinicians within the academic space and those in the community to address under-representation of AA and other minorities in clinical trials.

\section{Private practices}

For many years, most clinical trials were conducted in AMCs. However, in the last two decades, pharmaceutical companies have increasingly contracted with non-academic private-sector physicians. In 2010, 20250 US private-sector physicians were conducting clinical trials. ${ }^{75}$ This is about a fivefold increase from 1990, when just 4000 private-sectors physicians were involved. This significant growth can, in part, be attributed to an increase in the number of clinical trials and the physicians in private practice replacing academic physicians as principal investigators. ${ }^{75}$

The movement of clinical trials from academic spaces into the private sector can have negative consequences for the inclusion of minority participants in clinical trials for new therapies. In a landmark study, Bach $e t a l^{76}$ found that $22 \%$ of physicians provided $80 \%$ of the care to AA Medicare patients. Physicians visited by AA patients were less likely to be board-certified $(77.4 \%)$ compared with physicians visited by white patients $(86.1 \%, \mathrm{p}=0.02)$, and they were more likely to report difficulties in providing their patients with high-quality care $(27.8 \%$ vs $19.3 \%$, $\mathrm{p}=0.005) .{ }^{76}$ These findings are consistent with the results from a study by Varkey et $a l^{77}$ examining the impact of physician workplace conditions on disparities in healthcare. They reported that clinics serving at least 30\% minority patients have less access to referral specialists $(\mathrm{p}<0.005)$ and medical supplies $(\mathrm{p}<0.001)$, and there were fewer examination per physician $(\mathrm{p}=0.002) .{ }^{77}$ Additionally, clinics serving at least $30 \%$ minority patients were more likely to have chaotic work environments $(\mathrm{OR}=4.0$; $\mathrm{p}=0.01){ }^{77}$

Together, these studies suggest that as clinical trials move increasingly into the private sector, they tend 
to exclude minority physicians and minority patients. Clinicians evaluating primarily minority patients are less likely to be board-certified, ${ }^{76}$ impacting their likelihood to be considered as a principal investigator on a study. Additionally, their limited access to space and supplies compounded by a chaotic work environment ${ }^{77}$ makes these clinics unfavourable study sites.

\section{Clinical trials operation and regulation}

As one of the largest funders of clinical research, ${ }^{68}$ pharmaceutical companies have a major financial stake in the drug development process. Because delays in gaining Food and Drug Administration (FDA) approval can cost manufacturers a mean of $\$ 1.3$ million each day, ${ }^{68}$ there are significant incentives to execute the process as quickly as possible. On the other hand, companies are mindful of having postmarketing commitments based on poor enrolment, or other potential consequences of a hastily conducted trial. With the existing temporal and financial pressures, engaging $\mathrm{AA}$ and other minorities in clinical trials remains a significant challenge for pharmaceutical companies. ${ }^{33}$

In many instances, whether it be to conduct a trial efficiently or to enrol sufficient participants, pharmaceutical companies often seek the support of CROs. CROs, serving as intermediaries between pharmaceutical companies and clinical research sites, employ physician-scientists, biostatisticians, research coordinators and managers to offer a wide range of services to oversee the conduct of clinical trials. ${ }^{68}$ Pharmaceutical companies are opting more frequently to hire CROs to organise clinical trials. As major players in the for-profit market-place, evidence has suggested that CROs are able to complete trials rapidly and at a lower cost than those carried out by AMCs. ${ }^{78}$

CROs are able to achieve high efficiency, in part, because they develop a network of research sites and clinical investigators to execute trials rapidly. Once networks have been established and relationships are formed with experienced investigators, CROs will continue to use the same networks, which enhances their speed and ability to meet quality objectives ${ }^{50}$ Financial pressures and tight timetables dictated by industry sponsors leave CROs with little time to address other concerns, such as the appropriate representation of minority participants in clinical trials. Therefore, CROs often perform within the realm of what is convenient and efficient. In doing so, these organisations may indirectly exclude physicians who are caring for a large number of minority patients but may be lacking in research training or infrastructure, which exacerbates challenges in enrolling minority participants into trials. A possible solution could engage granting agencies, pharmaceutical sponsors and CROs to incorporate $\mathrm{racial} / \mathrm{ethnic}$ representation as an indicator of the quality of clinical trials.

If sufficient minority individuals do not participate in clinical trials, drug effects cannot be appropriately ascertained across various groups, and study results may not be generalisable to the larger population. Generalisability is critical in the drug development process because approved drugs should be effective and safe in all the populations that will consume them. The division of the FDA responsible for the approval of new drugs is the Center for Drug Evaluation and Research (CDER). CDER evaluates drugs to ensure that they are safe and efficacious, and that the benefits outweigh known risks, prior to marketing in the USA. $^{79}$

The approval of a pharmaceutical for marketing requires that several regulatory mandates are met. Of note, the FDA does not mandate pharmaceutical, biotechnology and medical device companies to include ethnic minorities in clinical trials. They provide guidance in a document titled 'Collection of Race and Ethnicity Data in Clinical Trials', which focuses on reporting race and ethnic data, but not on the importance of inclusion. Furthermore, this guidance is usually not enforced, and as a result about half of all trials accepted for review by the FDA fail to indicate any information on the race or ethnicity of study participants. ${ }^{50}$

As a regulatory agency, the FDA can mandate appropriate inclusion of minority participants to match the epidemiology of the disease, or to insist that such data are available before a drug is approved. However, their inconsistent enforcement of this mandate leaves stakeholders with little incentive to recruit minority patients for trials. Recently, the inability to show efficacy in minority subjects in the belimumab trials resulted in a postmarketing commitment to evaluate the agent in AA patients with lupus. Notably, however, this FDA-mandated postmarketing commitment trial known as the 'Efficacy and Safety of Belimumab in Black Race Patients With Systemic Lupus Erythematosus (EMBRACE)' has been recruiting AA participants since 2012. To date, the study has recruited about 500 participants across 120 sites internationally with a calculated recruitment rate of 0.07 patients per site per month at great expense to the sponsor, GlaxoSmithKline. ${ }^{80}$ Belimumab has remained available to AA patients with SLE pending the outcome of this trial. This experience emphasises the difficulty of recruiting AA subjects into lupus trials, but also shows the willingness of the regulatory agency to permit continuing marketing of the product to AA patients with lupus even without convincing evidence of benefit.

\section{CONCLUSION}

As the population becomes increasingly diverse, there is a growing necessity to ascertain drug effects across different ancestral backgrounds. For diseases, such as SLE, that disproportionately affect predominately minority individuals, the imperative is both scientific and ethical, as there are very few therapies for SLE that have been shown to benefit this segment of the population. An important step in developing more effective therapies is to recruit diverse study participants into clinical trials. For the many reasons discussed above, engaging minority patients in clinical research has proven challenging, but it is not 
impossible. Stakeholders have the ability to join forces to develop solutions to address this challenge. The most effective solutions will incentivise action beyond the status quo, or the development of a new status quo of inclusion.

Contributors AA and PL conceived of the project and wrote the manuscript. AA carried out the structured review.

Funding This study was funded by RILITE Foundation.

Competing interests None declared.

Patient consent Not required.

Provenance and peer review Not commissioned; externally peer reviewed.

Data sharing statement All data used in the preparation of this manuscript are in the public domain.

Open access This is an open access article distributed in accordance with the Creative Commons Attribution Non Commercial (CC BY-NC 4.0) license, which permits others to distribute, remix, adapt, build upon this work non-commercially, and license their derivative works on different terms, provided the original work is properly cited, appropriate credit is given, any changes made indicated, and the use is non-commercial. See: http://creativecommons.org/licenses/by-nc/4.0

\section{REFERENCES}

1. Wallace D, Hahn BH. Dubois' Lupus Erythematosus and Related Syndromes E-Book: Expert Consult-Online. Elsevier Health Sciences, 2012.

2. Pons-Estel GJ. NIH public access. , 2010: 39, 1-23.

3. Pons-Estel GJ, Alarcón GS, Scofield L, et al. Understanding the epidemiology and progression of systemic lupus erythematosus. Semin Arthritis Rheum 2010;39:257-68.

4. Murthy VH, Krumholz HM, Gross CP. Participation in cancer clinical trials. JAMA 2004;291:2720.

5. Heiat A, Gross CP, Krumholz HM. Representation of the elderly, women, and minorities in heart failure clinical trials. Arch Intern Med 2002;162:1682-8.

6. Witzig R. The medicalization of race: scientific legitimization of a flawed social construct. Ann Intern Med 1996;125:675-9.

7. Wilkinson R, Marmot M. Social determinants of health: the solid facts. World Health Organization, 2003.

8. Langefeld CD, Ainsworth HC, Cunninghame Graham DS, et al. Transancestral mapping and genetic load in systemic lupus erythematosus. Nat Commun 2017;8:16021.

9. Somers ECM, Cagnoli W, Lewis E P, et al. Population-based incidence and prevalence of systemic lupus erythematosus. Arthritis Rheumatol 2014;66:369-78.

10. Lim SS, Bayakly AR, Helmick CG, et al. The incidence and prevalence of systemic lupus erythematosus, 2002-2004: the Georgia Lupus Registry. Arthritis Rheumatol 2014;66:357-68.

11. Izmirly PM, Wan I, Sahl S, et al. The Incidence and prevalence of systemic lupus erythematosus in New York County (Manhattan), New York: The Manhattan Lupus Surveillance Program. Arthritis Rheumatol 2017;69:2006-17.

12. Dall'Era M, Cisternas MG, Snipes K, et al. The incidence and prevalence of systemic lupus erythematosus in San Francisco County, California: the California lupus surveillance project. Arthritis Rheumatol 2017;69:1996-2005.

13. Falasinnu $\mathrm{T}$, Chaichian $\mathrm{Y}$, Bass MB, et al. The Representation of gender and race/ethnic groups in randomized clinical trials of individuals with systemic lupus erythematosus. Curr Rheumatol Rep 2018;20:20.

14. Hanly JG, O'Keeffe AG, Su L, et al. The frequency and outcome of lupus nephritis: results from an international inception cohort study. Rheumatology 2016;55:252-62.

15. Feldman CH, Hiraki LT, Liu J, et al. Epidemiology and sociodemographics of systemic lupus erythematosus and lupus nephritis among US adults with Medicaid coverage, 2000-2004. Arthritis Rheum 2013;65:753-63.

16. Burgos PI, McGwin G, Pons-Estel GJ, et al. US patients of Hispanic and African ancestry develop lupus nephritis early in the disease course: data from LUMINA, a multiethnic US cohort (LUMINA LXXIV). Ann Rheum Dis 2011;70:393-4.

17. Richman IB, Taylor KE, Chung SA, et al. European genetic ancestry is associated with a decreased risk of lupus nephritis. Arthritis Rheum 2012;64:3374-82.
18. Plantinga L, Lim SS, Patzer R, et al. Incidence of end-stage renal disease among newly diagnosed systemic lupus erythematosus patients: the Georgia Lupus Registry. Arthritis Care Res 2016;68:357-65.

19. Fernández M, Alarcón GS, Calvo-Alén J, et al. A multiethnic, multicenter cohort of patients with systemic lupus erythematosus (SLE) as a model for the study of ethnic disparities in SLE. Arthritis Rheum 2007;57:576-84.

20. Korbet SM, Schwartz MM, Evans J, et al. Severe lupus nephritis: racial differences in presentation and outcome. J Am Soc Nephrol 2007;18:244-54.

21. Nee R, Martinez-Osorio J, Yuan CM, et al. Survival disparity of African American versus non-African American patients with ESRD due to SLE. Am J Kidney Dis 2015;66:630-7.

22. Gómez-Puerta JA, Feldman $\mathrm{CH}$, Alarcón GS, et al. Racial and ethnic differences in mortality and cardiovascular events among patients with end-stage renal disease due to lupus nephritis. Arthritis Care Res 2015;67:1453-62.

23. Barbhaiya M, Feldman $\mathrm{CH}$, Guan $\mathrm{H}$, et al. Race/Ethnicity and cardiovascular events among patients with systemic lupus erythematosus. Arthritis Rheumatol 2017;69:1823-31.

24. Rhew EY, Manzi SM, Dyer AR, et al. Differences in subclinical cardiovascular disease between African American and Caucasian women with systemic lupus erythematosus. Trans/ Res 2009;153:51-9.

25. Scalzi LV, Hollenbeak CS, Wang L. Racial disparities in age at time of cardiovascular events and cardiovascular-related death in patients with systemic lupus erythematosus. Arthritis Rheum 2010;62:2767-75.

26. González LA, Toloza SM, McGwin G, et al. Ethnicity in systemic lupus erythematosus (SLE): its influence on susceptibility and outcomes. Lupus 2013;22:1214-24.

27. Cooper GS, Parks CG, Treadwell EL, et al. Differences by race, sex and age in the clinical and immunologic features of recently diagnosed systemic lupus erythematosus patients in the southeastern United States. Lupus 2002;11:161-7.

28. Alarcón GS, McGwin G, Bartolucci AA, et al. Systemic lupus erythematosus in three ethnic groups. IX. Differences in damage accrual. Arthritis Rheum 2001;44:2797-806.

29. Bruce IN, O'Keeffe AG, Farewell V, et al. Factors associated with damage accrual in patients with systemic lupus erythematosus: results from the Systemic Lupus International Collaborating Clinics (SLICC) Inception Cohort. Ann Rheum Dis 2015;74:1706-13.

30. Cooper GS, Treadwell EL, St Clair EW, et al. Sociodemographic associations with early disease damage in patients with systemic lupus erythematosus. Arthritis Rheum 2007;57:993-9.

31. Dooley MA, Hogan S, Jennette C, et al. Cyclophosphamide therapy for lupus nephritis: poor renal survival in black Americans. Kidney Int 1997;51:1188-95.

32. Appel GB, Contreras G, Dooley MA, et al. Mycophenolate mofetil versus cyclophosphamide for induction treatment of lupus nephritis. J Am Soc Nephrol 2009;20:1103-12.

33. Navarra SV, Guzmán RM, Gallacher AE, et al. Efficacy and safety of belimumab in patients with active systemic lupus erythematosus: a randomised, placebo-controlled, phase 3 trial. Lancet 2011;377:721-31.

34. Furie R, Petri M, Zamani O, et al. A phase III, randomized, placebocontrolled study of belimumab, a monoclonal antibody that inhibits B lymphocyte stimulator, in patients with systemic lupus erythematosus. Arthritis Rheum 2011;63:3918-30.

35. Stohl W, Schwarting A, Okada M, et al. Efficacy and safety of subcutaneous belimumab in systemic lupus erythematosus: a fifty-two-week randomized, double-blind, placebo-controlled study. Arthritis Rheumatol 2017;69:1016-27.

36. Gómez-Puerta JA, Barbhaiya M, Guan H, et al. Racial/Ethnic variation in all-cause mortality among United States medicaid recipients with systemic lupus erythematosus: a Hispanic and asian paradox. Arthritis Rheumatol 2015;67:752-60.

37. Campbell R, Cooper GS, Gilkeson GS. Two aspects of the clinical and humanistic burden of systemic lupus erythematosus: mortality risk and quality of life early in the course of disease. Arthritis Rheum 2008;59:458-64.

38. Bernatsky S, Boivin JF, Joseph L, et al. Mortality in systemic lupus erythematosus. Arthritis Rheum 2006;54:2550-7.

39. Krishnan E, Hubert HB. Ethnicity and mortality from systemic lupus erythematosus in the US. Ann Rheum Dis 2006;65:1500-5.

40. Contreras G, Lenz O, Pardo V, et al. Outcomes in African Americans and Hispanics with lupus nephritis. Kidney Int 2006;69:1846-51.

41. Ward MM, Pyun E, Studenski S. Long-term survival in systemic lupus erythematosus. Patient characteristics associated with poorer outcomes. Arthritis Rheum 1995;38:274-83. 
42. Adler M, Chambers S, Edwards C, et al. An assessment of renal failure in an SLE cohort with special reference to ethnicity, over a 25year period. Rheumatology 2006;45:1144-7.

43. Feldman $\mathrm{CH}$, Hiraki LT, Winkelmayer WC. Serious infections among adult medicaid beneficiaries with systemic lupus erythematosus and lupus nephritis 2015;67:1577-85.

44. Dooley MA, Jayne D, Ginzler EM, et al. Mycophenolate versus azathioprine as maintenance therapy for lupus nephritis. N Engl $J$ Med 2011;365:1886-95

45. Ferucci ED, Johnston JM, Gaddy JR, et al. Prevalence and incidence of systemic lupus erythematosus in a population-based registry of American Indian and Alaska Native people, 2007-2009. Arthritis Rheumatol 2014;66:2494-502.

46. Sanders $P, 2011$. Dialogues on diversifying clinical trials. Available from: http://www.womenshealthresearch.org/site/PageServer? pagename=events_clinicaltrials

47. Hudson SV, Momperousse D, Leventhal H. Physician perspectives on cancer clinical trials and barriers to minority recruitment. Cancer Control 2005;12 Suppl 2:93-6.

48. Freimuth VS, Quinn SC, Thomas SB, et al. African Americans' views on research and the Tuskegee Syphilis Study. Soc Sci Med 2001;52:797-808.

49. George S, Duran N, Norris K. A systematic review of barriers and facilitators to minority research participation among African Americans, Latinos, Asian Americans, and Pacific Islanders. Am J Public Health 2014;104:e16-e31.

50. Agodoa L, Alanis AJ, Alexander-Bridges M, 2007. Increasing minority participation in clinical research. Heal Equity. Available from: http:// health-equity.pitt.edu/id/eprint/3209

51. Wendler D, Kington R, Madans J, et al. Are racial and ethnic minorities less willing to participate in health research? PLOS Med 2006;3:0201-10.

52. van Ryn M, Burgess D, Malat $\mathrm{J}$, et al. Physicians' perceptions of patients' social and behavioral characteristics and race disparities in treatment recommendations for men with coronary artery disease. Am J Public Health 2006;96:351-7.

53. Moskowitz D, Thom DH, Guzman D, et al. Is primary care providers' trust in socially marginalized patients affected by race? J Gen Intern Med 2011;26:846-51.

54. Moskowitz GB, Stone J, Childs A. Implicit stereotyping and medical decisions: unconscious stereotype activation in practitioners' thoughts about African Americans. Am J Public Health 2012;102:996-1001.

55. Sabin JA, Greenwald AG. The influence of implicit bias on treatment recommendations for 4 common pediatric conditions: pain, urinary tract infection, attention deficit hyperactivity disorder, and asthma. Am J Public Health 2012;102:988-95.

56. Sabin JA, Rivara FP, Greenwald AG. Physician implicit attitudes and stereotypes about race and quality of medical care. Med Care 2008;46:678-85.

57. Petri M, Perez-Gutthann S, Longenecker JC, et al. Morbidity of systemic lupus erythematosus: role of race and socioeconomic status. Am J Med 1991;91:345-53.

58. Howerton MW, Gibbons MC, Baffi CR, et al. Provider roles in the recruitment of underrepresented populations to cancer clinical trials. Cancer 2007;109:465-76.

59. Simon MS, Du W, Flaherty L, et al. Factors associated with breast cancer clinical trials participation and enrollment at a large academic medical center. J Clin Oncol 2004;22:2046-52.

60. Hardeman RR, Burgess D, Phelan S, et al. Medical student sociodemographic characteristics and attitudes toward patient centered care: do race, socioeconomic status and gender matter? A report from the Medical Student CHANGES study. Patient Educ Couns 2015;98:350-5

61. Cooper-Patrick L, Gallo JJ, Gonzales JJ, et al. Race, gender, and partnership in the patient-physician relationship. JAMA 1999;282:583-9.

62. Street RL, O'Malley KJ, Cooper LA, et al. Understanding concordance in patient-physician relationships: personal and ethnic dimensions of shared identity. Ann Fam Med 2008;6:198-205.

63. Betancourt JR. Cross-cultural medical education: conceptual approaches and frameworks for evaluation. Acad Med 2003;78:560-9.

64. Morgan SE, Occa A, Potter J, et al. "You Need to Be a Good Listener": recruiters' use of relational communication behaviors to enhance clinical trial and research study accrual. $J$ Health Commun 2017;22:95-101.

65. McCaskill-Stevens W, Pinto H, Marcus AC, et al. Recruiting minority cancer patients into cancer clinical trials: a pilot project involving the eastern cooperative oncology group and the national medical association. J Clin Oncol 1999;17:1029-39.

66. García JA, Paterniti DA, Romano PS, et al. Patient preferences for physician characteristics in university-based primary care clinics. Ethn Dis 2003;13:259-67.

67. Komaromy M, Grumbach K, Drake M, et al. The role of black and Hispanic physicians in providing health care for underserved populations. N Engl J Med 1996;334:1305-10.

68. Bodenheimer T. Uneasy alliance--clinical investigators and the pharmaceutical industry. N Engl J Med 2000;342:1539-44.

69. Mosley-Williams A, Lumley MA, Gillis M, et al. Barriers to treatment adherence among African American and white women with systemic lupus erythematosus. Arthritis Rheum 2002;47:630-8.

70. Ford JG, Howerton MW, Lai GY, et al. Barriers to recruiting underrepresented populations to cancer clinical trials: a systematic review. Cancer 2008;112:228-42.

71. Katz RV, Kegeles SS, Kressin NR, et al. The Tuskegee Legacy Project: willingness of minorities to participate in biomedical research. J Health Care Poor Underserved 2006;17:698-715.

72. Joseph G, Dohan D. Recruiting minorities where they receive care: Institutional barriers to cancer clinical trials recruitment in a safetynet hospital. Contemp Clin Trials 2009;30:552-9.

73. Jones L, Wells K. Strategies for academic and clinician engagement in community-participatory partnered research. JAMA 2007:297:407.

74. Thomas CR, Pinto HA, Roach M, et al. Participation in clinical trials: is it state-of-the-art treatment for African Americans and other people of color? J Natl Med Assoc 1994;86:177-82.

75. Fisher JA, Kalbaugh CA. United States private-sector physicians and pharmaceutical contract research: a qualitative study. PLOS Med 2012;9:e1001271.

76. Bach PB, Pham HH, Schrag D, et al. Primary care physicians who treat blacks and whites. N Engl J Med 2004;351:575-84.

77. Varkey AB, Manwell LB, Williams ES, et al. Separate and unequal: clinics where minority and nonminority patients receive primary care. Arch Intern Med 2009;169:243-50.

78. Fund C. From bench to bedside: preserving the research mission of academic health centers, 1999.

79. Ciociola AA, Cohen LB, Kulkarni P, et al. How drugs are developed and approved by the FDA: current process and future directions. Am $J$ Gastroenterol 2014;109:620-3.

80. ClinicalTrials.gov. Efficacy and Safety of Belimumab in Black Race Patients With Systemic Lupus Erythematosus (SLE) EMBRACE, 2012. 